\title{
Factores epizootiológicos de las estrongilosis gastrointestinales en cabras Criollas Cubanas: bases para un manejo integrado
}

Manuel Alejandro La O-Arias ${ }^{\text {a }}$

Francisco Guevara-Hernández ${ }^{\mathrm{b} *}$

Luis Alfredo Rodríguez- Larramendi ${ }^{\mathrm{c}}$

Luis Reyes-Muro ${ }^{\mathrm{d}}$

José Nahed-Toral $^{\text {e }}$

Hernán Orbelin Mandujano-Camacho ${ }^{\mathrm{f}}$

René Pinto-Ruiz ${ }^{\mathrm{g}}$

${ }^{a}$ Universidad Autónoma de Chiapas. Facultad de Ciencias Agronómicas Campus V. Chiapas. México.

b Universidad Autónoma de Chiapas. Facultad de Ciencias Agronómicas Campus V. Chiapas. México.

${ }^{c}$ Universidad de Ciencias y Artes de Chiapas (UNICACH). Facultad de Ingeniería. Chiapas. México.

d Instituto Nacional de Investigaciones Forestales, Agrícolas y Pecuarias (INIFAP), Aguascalientes, México.

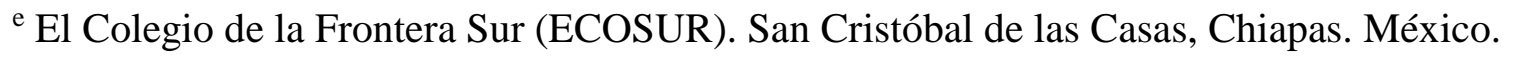

f Universidad Autónoma de Chiapas (UNACH). Facultad de Medicina, Veterinaria y Zootecnia. Chiapas. México.

g Universidad Autónoma de Chiapas (UNACH). Facultad de Ciencias Agronómicas Campus V. Chiapas. México.

*Autor de correspondencia: francisco.guevara@unach.mx 


\section{Resumen:}

El parasitismo causado por estrongilidos es uno de los principales factores limitantes de la producción de Cabras Criollas en el Este de Cuba. Mediante una investigación descriptiva y exploratoria realizada durante el periodo comprendido entre los años 2013 y 2018, fueron identificados los factores que regulan la dinámica epizootiológica de las estrongilosis gastrointestinales en 18 rebaños. Los factores bajo control fueron: la dinámica de población de larvas por mes, los estados reproductivos y el proceso de crecimiento de las cabras. Se registró la dinámica mensual de larvas infestivas en el pasto. Se aplicaron ANOVAS simples para modelos lineales correspondientes a cada factor y la prueba de Newmankeuls para comparaciones múltiples de media. Se observó que las dinámicas parasitarias de las estrongilosis gastrointestinales, que afectan a las cabras Criollas Cubanas, están relacionadas con procesos fisiológicos y zootécnicos. En estas dinámicas se identificaron dos momentos críticos o picos de infestación: en los animales en crecimiento durante el periodo de destete (2,188 huevos por gramo, HPG) y en las reproductoras en periodos de periparto (972 HPG). La infestación general de los rebaños está condicionada por la combinación de mayor ingestión de larvas infestivas, procesos de estrés alimentario y estados fisiológicos predisponentes, que conducen a picos de infestación estacionales entre los meses de diciembre a febrero superiores a 1,500 HPG. La dinámica de infestación del pasto está relacionada con la estacionalidad lluviosa con picos de infestación entre los meses de julio a septiembre y un máximo promedio de 1,200 larvas por kilo de pasto.

Palabras clave: Caprino, Infestación parasitaria, Hospedero, Pasto.

Recibido: 08/02/2020

Aceptado.05/02/2021

\section{Introducción}

Las cabras Criollas Cubanas son un genotipo diferenciado de sus ancestros ibéricos y africanos $^{(1)}$. Esta diferenciación es el resultado de 500 años de coevolución en el contexto socio ambiental del Este de Cuba, región donde se concentra más del $90 \%$ de la población caprina cubana y donde se registró oficialmente el primer grupo de animales de esta raza ${ }^{(2)}$. Según La $\mathrm{O}$ et $a l^{(3)}$ estos animales son propios de sistemas de crianza campesinos, fundamentalmente con objetivos de autosubsistencia. Por esta razón, sus criadores destinan muy pocos insumos para esta actividad y esperan tener sus producciones sobre la base de la 
rusticidad del genotipo. Sin embargo, otros investigadores encontraron que estos animales podían portar cargas significativas de estrongilidos gastrointestinales e informaron, incluso, la presencia de dos especies de Haemonchus, contortus y placei, esta última, de alta especificidad para el ganado bovino ${ }^{(4)}$.

Varios estudios sobre el efecto de este grupo parasitario han demostrado que pueden afectar más del $25 \%$ del potencial productivo de los animales, sin mostrar señales para los productores ${ }^{(5)}$. De esta forma, se estima que el parasitismo causado por estrongilidos es uno de los principales factores limitantes de la producción de Cabras Criollas en el Este de Cuba. Esta situación genera polémica sobre las estrategias más convenientes para el desarrollo de los sistemas tradicionales de crianza. Los esquemas convencionales de tratamientos antihelmínticos no son compatibles con la racionalidad tradicional de crianza, por esta razón todo apunta a desarrollar estrategias de control integrado más consistentes con la visión del criador campesino.

Para generar estrategias de control integrado, es importante identificar las particularidades del proceso parasitario para estas cabras en la región. De esta problemática se genera la pregunta de investigación que dio lugar al presente estudio ¿Cuáles son los factores que definen la dinámica de las estrongilosis gastrointestinales que afectan a las cabras Criollas Cubanas? Entonces, el objetivo fue identificar los factores que regulan la dinámica de las estrongilosis gastrointestinales de las cabras Criollas Cubanas.

\section{Material y métodos}

\section{Ubicación del estudio y muestra}

La investigación se realizó en la subcuenca de los ríos Cautillo-Jiguaní, del Valle del Río Cauto, ubicada en el este de Cuba, Municipio de Jiguaní, provincia de Granma (Figura 1) Los rebaños pertenecían a la comunidad " 26 de Julio", zona donde se registraron por primera vez en Cuba, ejemplares de esta raza como raciales puros. 
Figura 1: Ubicación del área de estudio

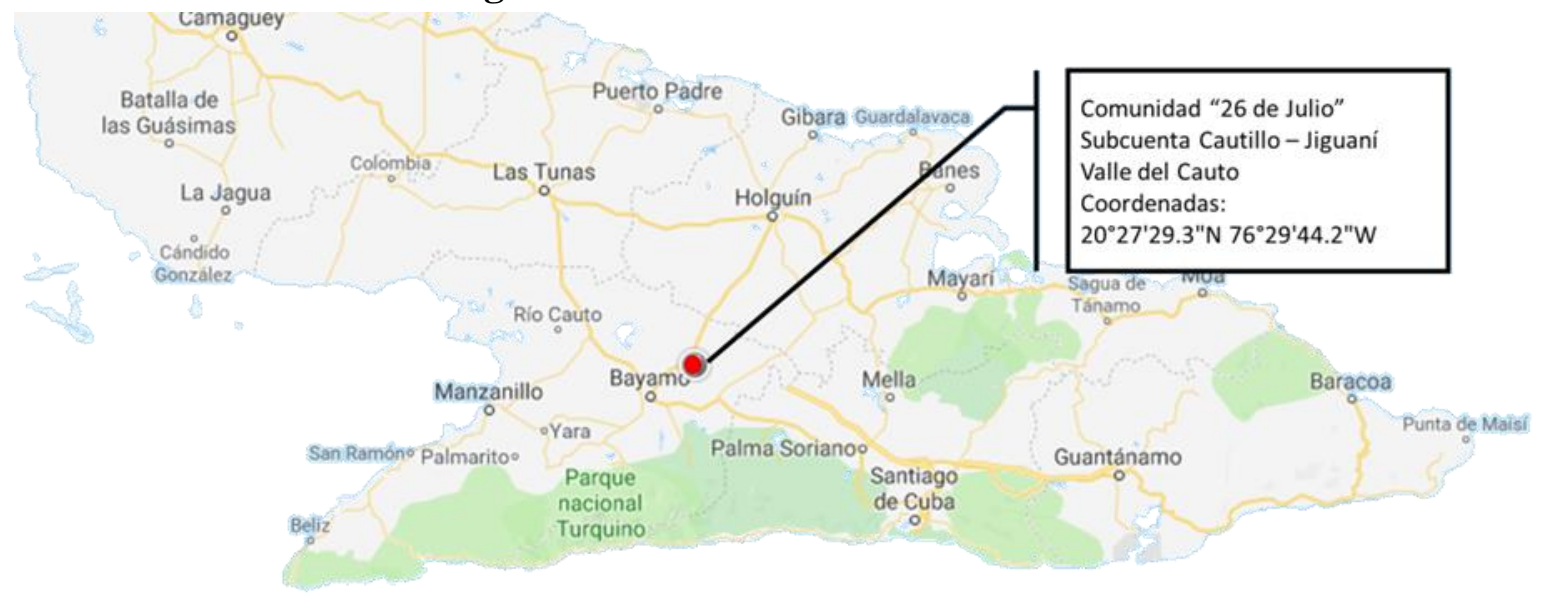

Se seleccionaron 18 rebaños en correspondencia con las tipologías de crianza tradicionales para esta raza, identificadas por $\mathrm{La} \mathrm{O}$ et $a l^{(3)}$. En total se investigaron 860 animales, de ellos: 26 sementales, 455 reproductoras y 379 animales en crecimiento. Los animales fueron apreciados por expertos y clasificados como Criollos Cubanos.

\section{Diseño de la investigación}

La investigación realizada fue del tipo descriptiva y exploratoria. El objeto de estudio fue el proceso parasitario por medio de dos variables o indicadores: 1) el número de huevos de parásitos por gramo de heces (HPG) y el número de larvas infestivas en el pasto (larvas por kilo de pasto). Estas variables se monitorearon por tres años con frecuencia mensual.

En el caso de los hospederos, se tomó una muestra mínima de 10 animales por rebaño pero que en su conjunto representara más del $10 \%$ de los animales existentes por categorías. En total se trabajaron 3,715 muestras de heces fecales. En el caso de los pastos, las muestras se tomaron del área en ocupación y de las áreas con menos de 28 días de descanso. En total se procesaron 1,250 muestras de pastos procedentes de las 18 unidades de crianza.

Los factores de influencia epizootiológica controlados fueron: a) mes del año; b) categoría de crianza (sementales, reproductoras y animales en crecimiento hasta el destete (120 días) y después del destete); c) estado fisiológico (animales secos y vacíos, tiempo de gestación y tiempo de lactancia) 


\section{Estudios parasitológicos}

Las muestras de heces fecales se tomaron antes de las $0800 \mathrm{~h}$ con frecuencia mensual. Para identificar los géneros presentes se realizaron cultivos por siete días protegidos con carbón activado. El conteo de huevos se realizó mediante la técnica de Mac Master ${ }^{(6)}$. En el caso de los pastos la muestra se tomó antes de las $0800 \mathrm{~h}$, mediante un recorrido por las áreas de pastoreo en el cual se recogieron pequeñas porciones de pasto cada 10 pasos. Para el conteo de larvas infestivas por kilo de pasto se utilizó la técnica descrita por Arece y Rodríguez ${ }^{(7)}$.

\section{Análisis estadístico}

Se aplicaron ANOVAS simples para modelos lineales correspondientes con cada uno de los factores controlados (periodo de crecimiento, mes, estados reproductivos). Para las comparaciones múltiples de media se aplicó la prueba de Newmankeuls. El software utilizado fue STATISTICA $12.0^{(8)}$.

\section{Resultados y discusión}

Los géneros de parásitos diagnosticados en los cultivos de larvas fueron Haemonchus (48 \%), Bunostomun (23\%), Trichostrongylus (23\%) y Oesophagostomun (6\%). Esta estructura poblacional concordó con los resultados informados por Rojas et $a l^{(4)}$ en esta misma localidad. En realidad, es consistente con un patrón de estructura poblacional característico de las zonas tropicales ${ }^{(9)}$. El predominio del género Haemonchus está dado por las condiciones favorables para desarrollar su fase exógena durante todo el año y porque se trata de un grupo mucho más prolífero que el resto de los géneros identificados ${ }^{(10)}$. Los géneros Haemonchus y Oesophagostomum predominan a temperatura y humedad elevadas, mientras que el género Cooperia lo hace en climas húmedos y tropicales a la inversa de Teladorsagia que incide en climas templados o fríos ${ }^{(11)}$.

Para la dinámica de expulsión de huevos durante el ciclo reproductivo (Figura 2), se encontró un incremento durante el periodo de periparto; fenómeno epizootiológico denominado PPR (siglas en inglés) descrito por varios autores ${ }^{(12-13)}$; los que refieren que, durante este periodo, que incluye un mes antes y un mes después del parto, se producen determinados procesos inmunodepresores que propician el desarrollo de larvas que se habían mantenido hipobióticas. 
Figura 2: Dinámica de la expulsión de huevos en cabras durante el ciclo reproductivo

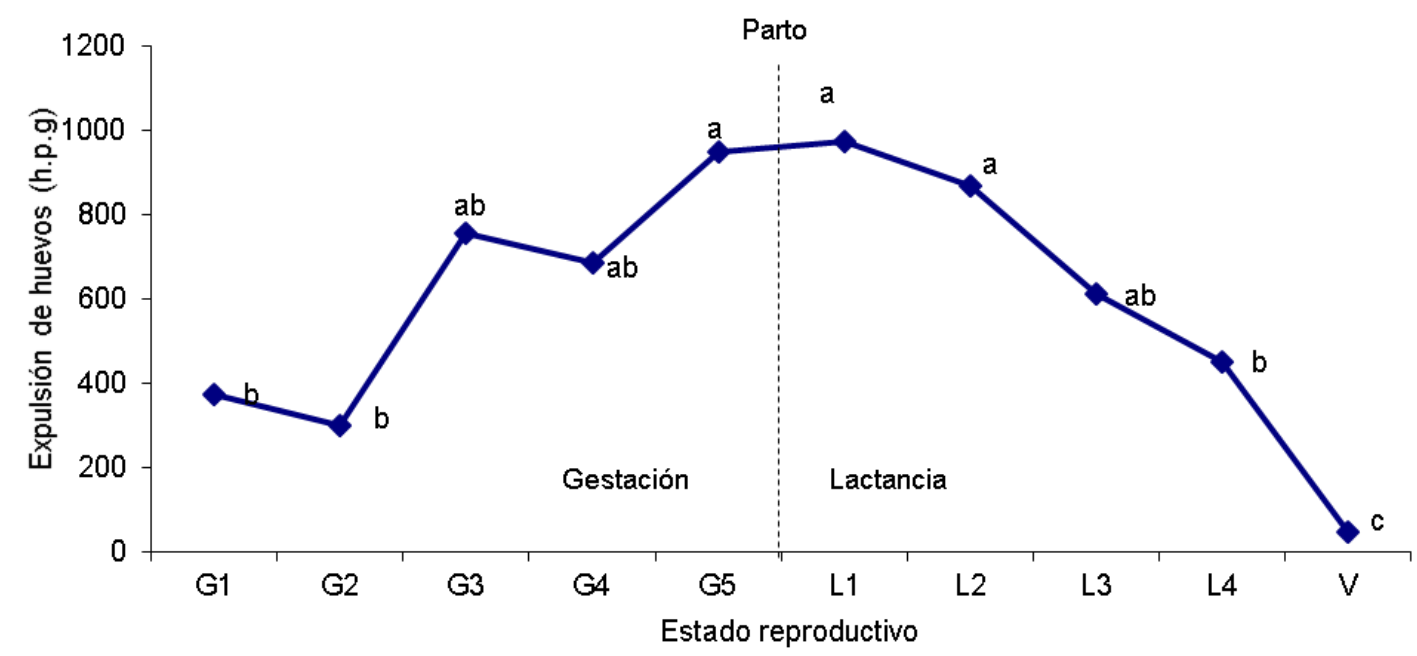

G1 a G5= gestante de 1 a 5 meses; L1 a L4= lactante de 1 a 4 meses; V= vacía; abc $P<0.01 ; \mathrm{CV}=12 \%$.

Tampoco se descarta la posibilidad de incrementos de la fertilidad de hembras de parásitos con mayores producciones de huevos. En la Figura 3 se observan dos fotos de hembras de Haemonchus spp., en la sección cercana a las aletas vulvares. En estas imágenes, se puede apreciar mayor carga aparente de huevos en la hembra de parásitos, recuperada de una reproductora caprina en periparto. Esta no es una evidencia concluyente, pero induce a la investigación para probar esta hipótesis.

Figura 3: Secciones de aletas vulvares de hembras de Haemonchus spp., recuperadas de reproductoras caprinas dentro y fuera del periodo de periparto (Fotos: Manuel A. La O Arias)

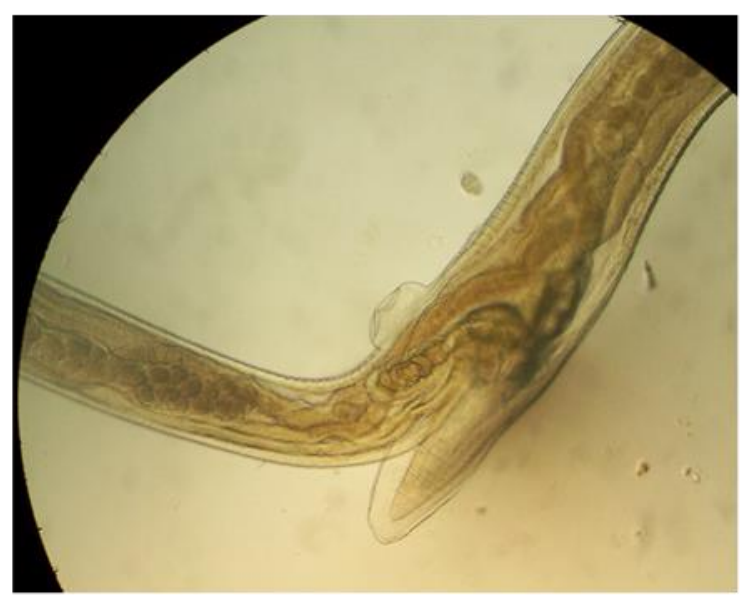

Hembra de Haemonchus spp., recuperada de una reproductora caprina no gestante Aleta vulvar lingüiforme.

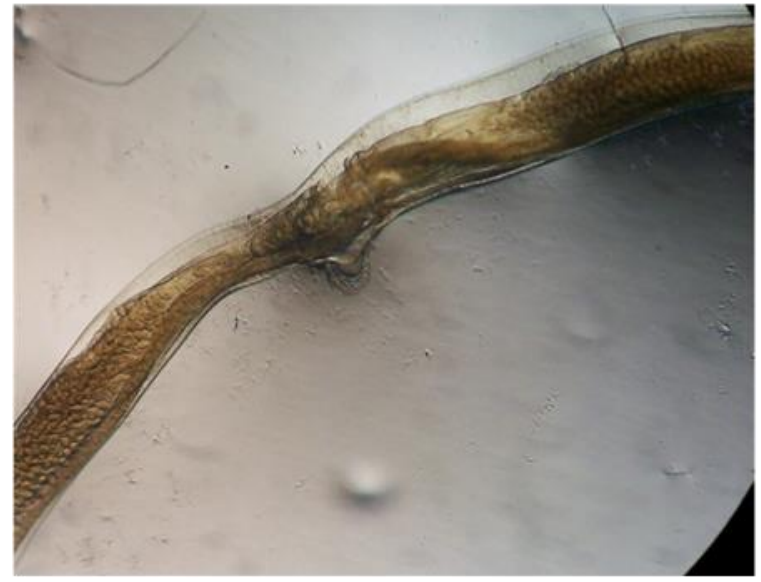

Hembra de Haemonchus spp., recuperada de una reproductora caprina con 120 días de gestación. Aleta vulvar botonosa. 
En Cuba, el primer informe de procesos de incremento de la actividad parasitaria en el periodo de periparto fue realizado en la especie ovina ${ }^{(14)}$. En esta investigación, se realiza el primer informe del proceso de periparto en la especie caprina en Cuba.

Respecto a la dinámica de la expulsión de huevos durante el periodo de crecimiento (Figura 4), se encontró que la infestación de las crías comienza a manifestarse poco antes de los 60 días y muestra una tendencia a incrementarse hasta alcanzar picos significativos $(P<0.001)$ entre los 90 y 120 días (más de 1,800 huevos por gramo (HPG). Este periodo coincide con la caída del consumo de leche, ya sea por destete o por reducción significativa de la producción de la madre. A partir de ese momento (120 a 135 días) se reduce significativamente la infestación hasta alcanzar los niveles más bajos a los 180 días de edad con picos esporádicos.

Figura 4: Dinámica de la expulsión de huevos de parásitos en cabritos criollos en crecimiento

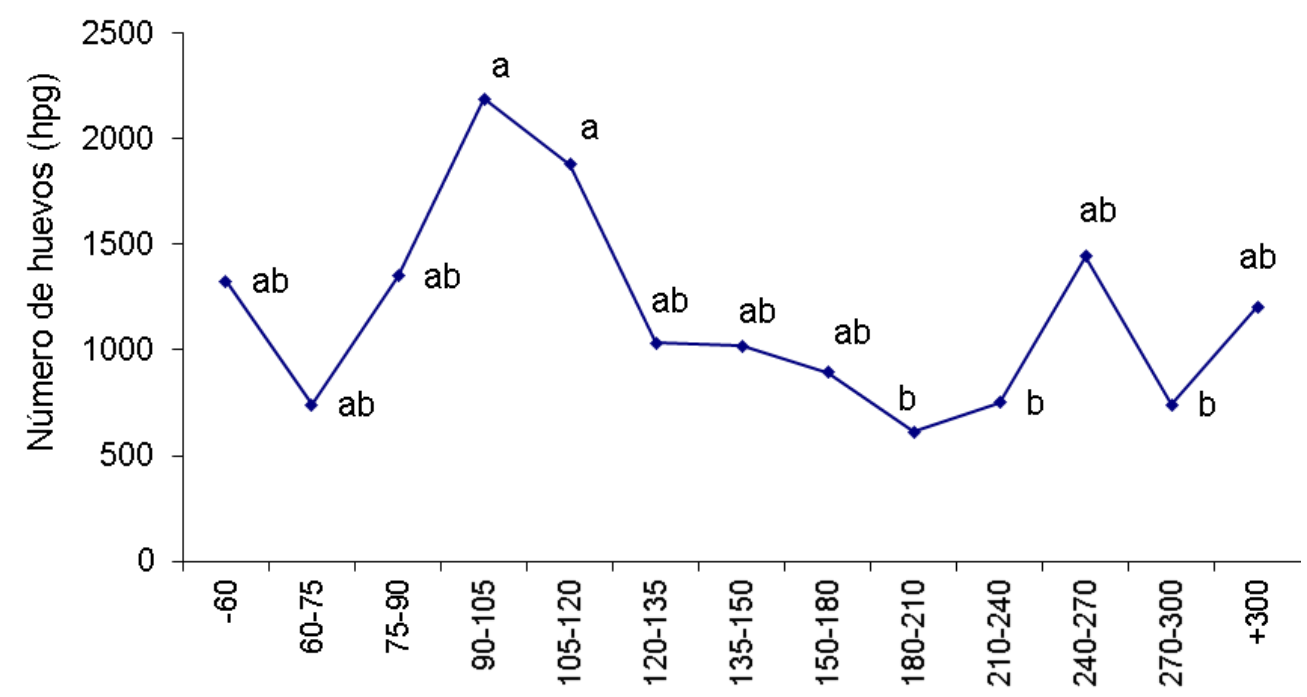

Periodo de crecimiento (días)

ab $P<0.10 ; \mathrm{CV}=20 \%$.

El incremento de la infestación en las crías alrededor del destete ha sido reportado por diferentes autores ${ }^{(15-16)}$, los que coinciden en el planteamiento de que las crías tienen un sistema inmunológico inmaduro a lo cual se agrega el estrés del destete. Cuando se reduce o se trunca bruscamente el consumo de leche se incrementa el consumo de pasto, por tanto, se incrementa el consumo de larvas infestivas. En ese periodo, comienzan a activarse los mecanismos de respuesta de inmunidad activa en los animales en crecimiento ${ }^{(17)}$. En cuanto a la reducción del conteo a partir de los 180 días, ocurre el proceso denominado de "auto cura" en ovinos, el cual se verifica alrededor de este mismo periodo (180 días). Este proceso tiene bases inmunológicas y su continuidad estará determinada por factores que alteran el estado de resistencia general del individuo hospedero ${ }^{(18)}$. 
La dinámica mensual de la expulsión de huevos en las heces (HPG) en las reproductoras (Figura 5) mostró que el nivel de infestación presentó una tendencia a incrementarse a partir de octubre. El pico de infestación se presentó en diciembre (1,722 HPG), lo que denota un mayor nivel de infestación durante los meses secos.

Figura 5: Dinámica mensual del conteo de huevos de parásitos en las heces fecales en reproductoras caprinas Criollas

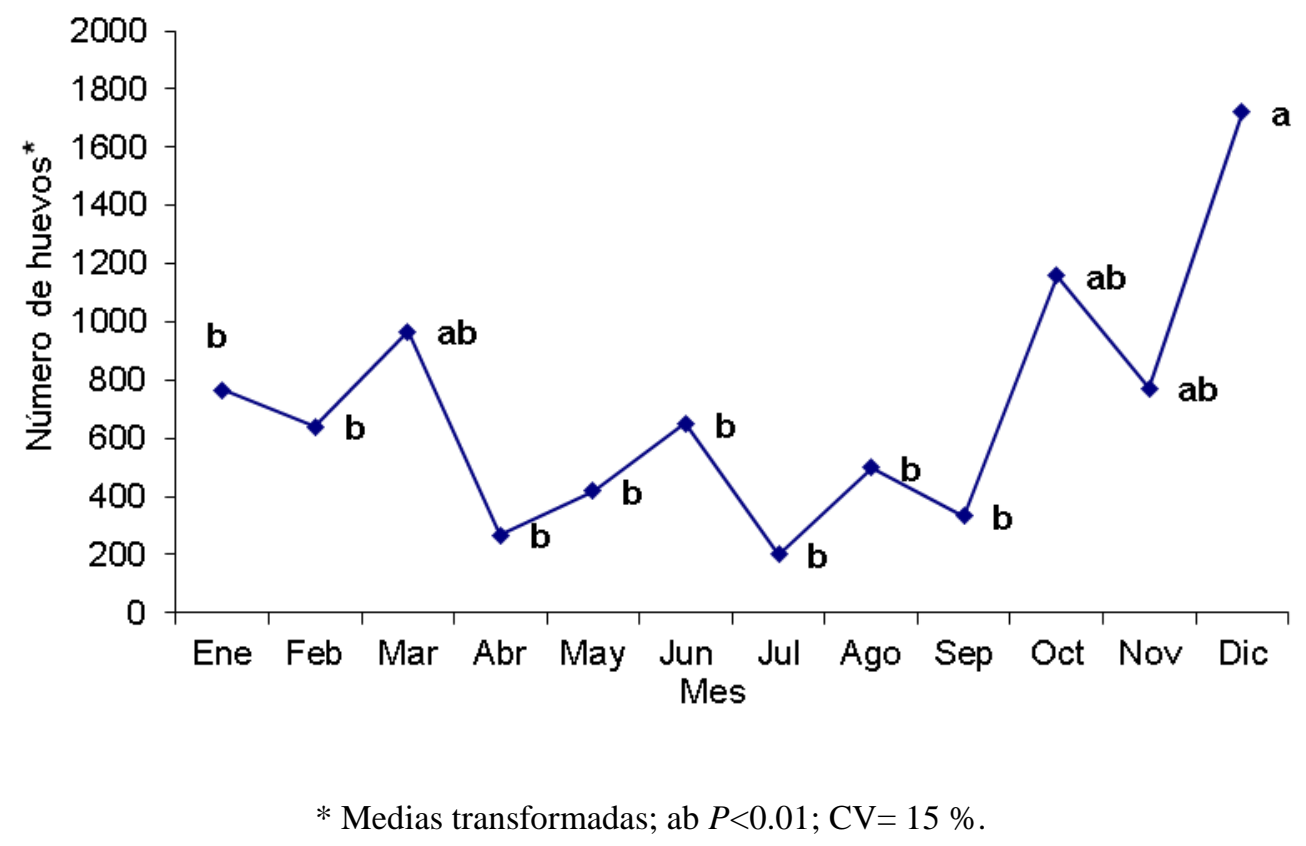

La dinámica mensual del conteo de huevos en animales en crecimiento (Figura 6), mostró los niveles más altos en diciembre, enero y febrero $(P<0.001)$, que coincide con el periodo seco y con las temperaturas más bajas. En marzo y abril comienza a bajar la infestación, con un nuevo pico más ligero en mayo (inicio del periodo lluvioso). Posteriormente, la infestación desciende hasta alcanzar los niveles más bajos entre julio y octubre. A partir de noviembre (inicio del periodo seco), comienza a incrementarse nuevamente. 
Figura 6: Dinámica mensual del conteo en huevos en cabras Criollas Cubanas en crecimiento

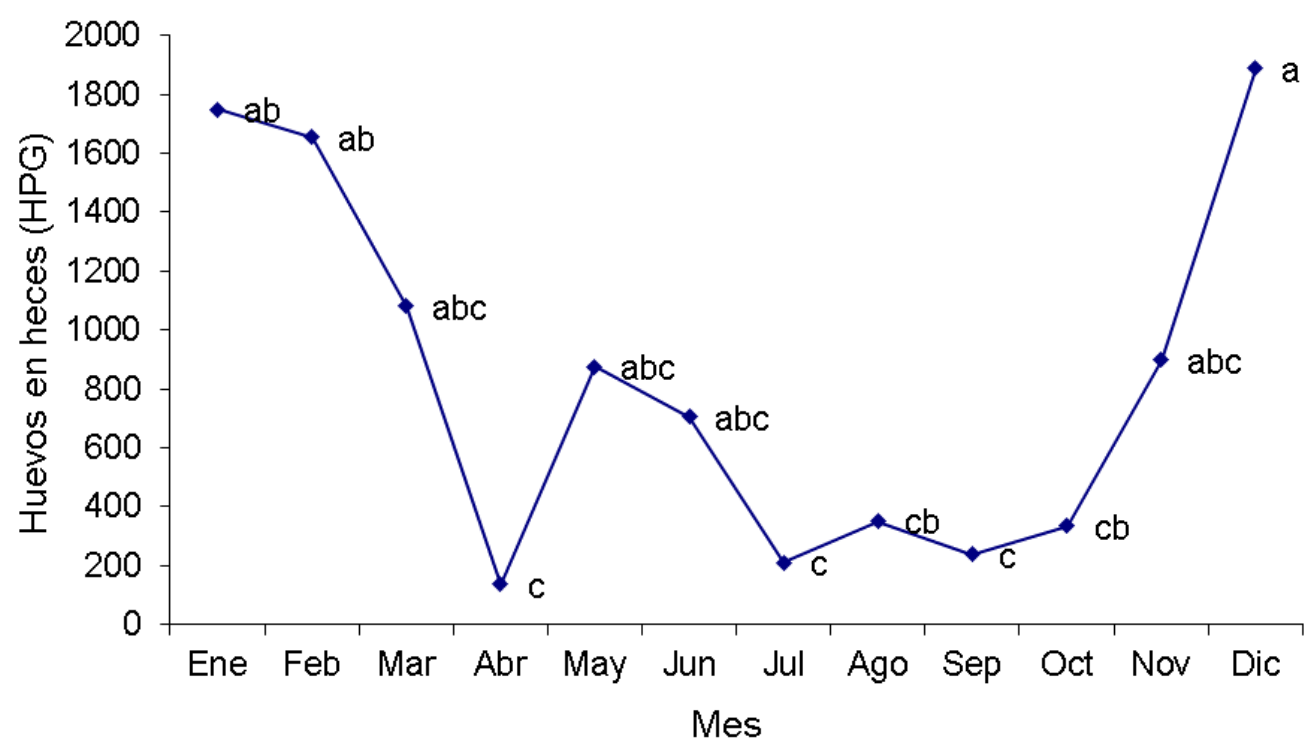

Bedotti et $a l^{(11)}$ explicaron que, en el periodo seco, las condiciones son menos propicias para el desarrollo de la fase exógena del ciclo biológico de estos parásitos, debido a la combinación de altas temperaturas y baja humedad, letal para las larvas pre-infestivas. Diversos estudios realizados en condiciones tropicales en especies menores muestran mayores infestaciones durante la lluvia $^{(19)}$. Este comportamiento, en los sistemas tradicionales de crianza de Cabras Criollas, en Cuba, se ve reforzado por la ausencia de prácticas de suplementación alimentaria en el periodo seco. Las cargas de animales en las áreas de pastos se incrementan en función de la disponibilidad de alimentos ${ }^{(1)}$. Entonces, se produce una caída del estado de resistencia de los hospederos por estrés alimentario y las estrongilosis gastrointestinales, comienzan a presentarse con más intensidad. En este caso, los parásitos encuentran hospederos más susceptibles que les brindan mejores condiciones para el desarrollo de la fase endógena de su ciclo biológico. Además del estrés alimentario, el consumo a fondo de los pastos y niveles favorables de humedad, crean condiciones propicias para que el rebaño consuma mayor cantidad de larvas infestivas. La humedad favorece los procesos de desarrollo y migración de larvas parasitarias.

La Figura 7 muestra la dinámica mensual de la población de larvas infestivas en el pasto. Los niveles de infestación más altos se presentaron en el periodo de julio a noviembre. Estos periodos de máxima contaminación larvaria coinciden con la época lluviosa, donde las condiciones climáticas favorecen el desarrollo de las larvas. 
Figura 7: Comportamiento de la dinámica mensual de larvas infestivas en el pasto en sistemas de crianza de cabras Criollas Cubanas

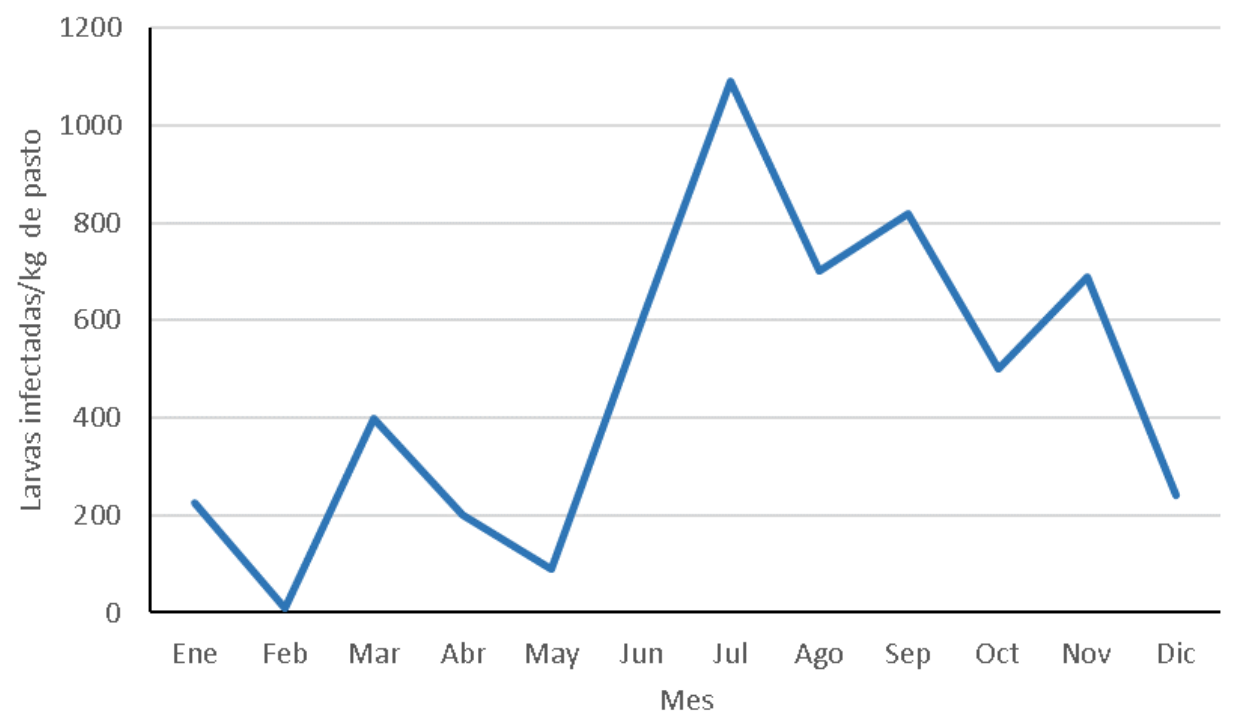

Durante la fase exógena, las condiciones de humedad, temperaturas y radiación definen el éxito del desarrollo de las larvas. Por tanto, las variaciones estacionales de estos parámetros ambientales son generalmente un factor determinante en el comportamiento epidemiológico de las estrongilosis gastrointestinales. Según Bedotti et $a l^{(11)}$ las altas temperaturas en el período estival causan mortandad de las larvas infectivas, lo que merma la población de nemátodos. Temperaturas elevadas, por encima de 35 a $40{ }^{\circ} \mathrm{C}$ se presentan como el segundo factor de importancia con una acción letal sobre los estadios preinfestantes.

En el área de estudio, las temperaturas máximas anuales oscilan entre 29 y $33{ }^{\circ} \mathrm{C}$, mientras, las mínimas promedio oscilan entre 17 y $22{ }^{\circ} \mathrm{C}^{(20)}$. Este intervalo de temperaturas es óptimo para el desarrollo larval en el pasto durante todo el año. Entonces, la estacionalidad descrita en este estudio está determinada por diversas interacciones entre la estacionalidad de las lluvias y los estados fisiológicos de los hospedadores.

En el Valle del Cauto existen dos periodos climáticos bien definidos: 1) el periodo lluvioso, de mayo a octubre y 2) el periodo seco, de noviembre a abril. Ramírez et al ${ }^{(20)}$ describieron las variaciones en la producción de biomasa asociadas a estas épocas lo que explica la dinámica larvaria del pasto. Sin embargo, la dinámica de infestación en el hospedero sigue a la combinación de procesos más complejos. En las reproductoras, se combina el efecto de estrés alimentario, en periodo seco, con el estrés fisiológico del periparto. Esta combinación se refleja en el pico de infestación identificado en el mes de diciembre, donde se concentran los $\operatorname{partos}^{(1)}$ y se agudiza el estrés alimentario por la poca producción de biomasa que caracteriza ese mes. En el caso de los animales en crecimiento, también se verifica este pico por estrés alimentario en periodo seco, pero, además, se observa un pico menos marcado en 
el mes de mayo. Este comportamiento en epizootiología del parasitismo gastrointestinal se conoce como "alza de primavera".

El "alza de primavera" ya había sido informada en condiciones de montaña, en el Este de $\mathrm{Cuba}^{(21)}$. Aunque es necesario seguir estudiando este aspecto de la dinámica mensual, se puede afirmar que en ambos casos hay tres puntos de coincidencia que ayudan a explicar las causas de este pico. Primero, en este periodo se concentran destetes, lo que significa muchos animales estresados. Segundo, las áreas de pastoreo están altamente contaminadas por la concentración de hembras en periparto que depositan heces con altos valores de HPG. Tercero, el comienzo de las lluvias facilita la dilución de las heces y los procesos migratorios de las larvas infestivas acumuladas en el último mes del periodo seco.

\section{Conclusiones e implicaciones}

Las dinámicas parasitarias de las estrongilosis gastrointestinales, que afectan a las cabras Criollas Cubanas, están relacionadas con procesos fisiológicos y zootécnicos que definen momentos críticos en los animales en crecimiento durante el periodo de destete (2,188 HPG) y para las reproductoras en periodos de periparto (972 HPG). La infestación general de los rebaños está condicionada por la combinación de mayor ingestión de larvas infestivas, procesos de estrés alimentario y estados fisiológicos predisponentes, que condicionan picos de infestación entre los meses de diciembre a febrero superiores a 1,500 HPG. La dinámica de infestación del pasto está relacionada con la estacionalidad lluviosa con picos de infestación entre los meses de julio a septiembre con un máximo promedio de 1,200 larvas por kilo de pasto.

\section{Conflictos de interés}

Los autores declaran no haber recibido fondo alguno para la realización de la presente investigación. Tampoco existe conflicto de interés entre los autores y la revista o alguna otra instancia o institución relacionada con la presente investigación. Este trabajo debido al tipo de investigación realizada, no presenta ninguna implicación ética o bioética. 


\section{Literatura citada:}

1. Chacón ME, La O AM, Fonseca FN, Pérez PE, Velázquez RFJ, Cos DY, Fonseca JY, et al. Caracterización genética y conservación de la Cabra Criolla Cubana. En: Biodiversidad caprina iberoamericana. Vargas-Bayona JE. (Comp.). Bogotá: Ed. Universidad Cooperativa de Colombia, 2016:75-86.

2. CENCOP. Centro de Control Pecuario. Registros de razas puras y ferias ganaderas. Ministerio de la Agricultura. Granma. Cuba. 2018.

3. La O AM, Guevara HF, Rodríguez LLA, Pinto RR, Nahed TJ. Ley de CA, Reyes ML. Evolución de los sistemas de crianza de Cabras Criollas Cubanas en el contexto de la conservación del genotipo. Rev Mex Cienc Pecu 2018;9(1):68-85.

4. Rojas N, La O AM, Arece J, Carrión M, Pérez K, San-Martín C, Valerino P, et al. Identificación y caracterización de especies de haemonchus en caprinos del Valle del Cauto en Granma. REDVET. Revista Electrónica de Veterinaria 2012;13(2):1-10. http://www.redalyc.org/articulo.oa?id=63623405003._Consultado: 25 Sep, 2019.

5. Parra RI, Magaña MA, Duarte JH, Téllez G. Caracterización técnica y rentabilidad de granjas ovinas con visión empresarial del departamento del Tolima. Rev Colombiana Cienc Anim 2014;7(1):64-72.

6. Witlock JH. The evaluation of pathological growth and parasitic diseases. Cornell Vet 1955;(45):411-421.

7. Arece J, Rodríguez JG. Dinámica de las larvas infestantes de estrongílidos gastrointestinales en ovinos en pastoreo. Pastos y Forrajes 2010;33(1):1-17.

8. StatSoft, Inc. STATISTICA (Data Analysis Software System), version 12.0., 2017 www.statsoft.com.

9. Figueroa AA, Pineda RSA, Godínez JF, Vargas ÁD, Rodríguez BE. Parásitos gastrointestinales de ganado bovino y caprino en Quechultenango, Guerrero, México. Agroproductividad 2018;11(6):97-104.

10. Rossanigo C, Page W. Evaluación de FAMACHA en el control de nematodos gastrointestinales en cabras de San Luis (Argentina). RIA. Rev Invest Agropec 2017;43(3):239-246.

11. Bedotti DO, Cristel SL, Lux J M, Hurtado AW, Babinec FJ. Presencia y dinámica parasitaria en dos majadas de Cabras Criollas en el oeste de la Provincia de la Pampa, Argentina. Actas Iberoamericanas de Conservación Animal AICA 2018;12:164-170. 
12. da Rosa FR, Leite TE, Mendes ADD. Correlação entre condição corporal e parasitismo de ovelhas no periparto e o desenvolvimento dos cordeiros. Anais do 10 Salão Internacional de Ensino, Pesquisa e Extensão 2018;9(3). https://guri.unipampa.edu.br/uploads/evt/arq_trabalhos/13079/seer_13079.pdf. Consultado: 25 Sep, 2019.

13. Silva JB, Castro GNS, Fonseca AH. Comparação da prevalência de parasitos gastrointestinais em cabras mantidas em manejo orgânico e convencional. Revista de Educação Continuada em Medicina Veterinária e Zootecnia do CRMV-SP 2013;11(1):69-69.

14. Arece J, Rodríguez DJG, López Y. La metodología FAMACHA®: una estrategia para el control de estrongilidos gastrointestinales de ovinos. Estudios preliminares. Rev Salud Anim 2007;29(2):91-94.

15. Suárez VH, Rossanigo CE, Descarga C. Epidemiologia e impacto productivo de nematodos en la Pampa Central de Argentina. Enfermedades parasitarias de importancia clínica y productiva en rumiantes. Fundamentos epidemiológicos para su diagnóstico y control. Eds. Fiel 2013;C:59-88.

16. Arece J. El control integrado del parasitismo gastrointestinal en los rumiantes: la garantía de un rebaño sano. Pastos y Forrajes 2012;23(1): https://payfo.ihatuey.cu/index.php?journal=pasto\&page=article\&op=view\&path\%5B \%5D=959. Consultado: 25 Sep, 2019.

17. Charlier J, Morgan ER, Rinaldi L, Van Dijk J, Demeler J, Höglund J, Kenyon F. Practices to optimize gastrointestinal nematode control on sheep, goat and cattle farms in Europe using targeted (selective) treatments. Vet Record 2014;175(10):250-255.

18. Suárez VH, Fondraz M, Viñabal AE, Salatin AO. Validación del método FAMACHA@ para detectar anemia en caprinos lecheros en los valles templados del Noroeste Argentino. Rev Med Vet 2014;95(2):4-11.

19. de Macedo FD, Lorenço FJ, Santello GA, Martins EN, de Moraes GV, Mexia AA, Mora NHAP. Parasitose gastrointestinal e valor do hematócrito em fêmeas ovinas alimentadas com diferentes níveis de proteína bruta. Rev Ciênc Agroamb 2016;13(2):65-73.

20. Ramírez JL, Herrera RS, Leonard I, Verdecia D, Álvarez Y. Rendimiento de materia seca y calidad nutritiva del pasto Brachiaria brizantha x Brachairia ruziziensis cv. Mulato en el Valle del Cauto, Cuba. Rev Cubana Cienc Agr 2010;44(1):65-72.

21. La O M, Fonseca N, Costa PJ, Carrión M, Vázquez J, Liranza, E, García, A. Infestación por nematodos gastrointestinales en un sistema de explotación caprina silvopastoril en condiciones de montaña. Pastos y Forrajes 2003;26(1):53-59. 